\title{
Pressão arterial elevada em adolescentes: prevalência e fatores associados
}

\author{
High blood pressure in adolescents: \\ prevalence and associated factors
}

Diego Augusto Santos Silva ${ }^{1}$

Luiz Rodrigo Augustemak de Lima ${ }^{1}$

Rodolfo André Dellagrana ${ }^{1}$

Eliane Denise Araújo Bacil ${ }^{2}$

Cassiano Ricardo Rech ${ }^{1}$

\footnotetext{
${ }^{1}$ Programa de Pós-

Graduação em Educação Física, Departamento de Educação Física, Universidade Federal de Santa Catarina. Campus Universitário Trindade, Caixa Postal 476, Trindade. 88.010-970 Florianópolis Santa Catarina Brasil. diegoaugustoss@yahoo.com.br ${ }^{2}$ Universidade Federal do Paraná. Programa de PósGraduação em Educação Física.
}

\begin{abstract}
The aim of this study was to assess the prevalence and associated factors of high Blood Pressure (BP) among adolescents in a Brazilian city. A cross-sectional study was conducted with 653 adolescents (57.9\% female) of high school level between 14 and 19 years of age. Systolic blood pressure (SBP) and diastolic blood pressure (DBP) of all subjects were measured. Social and demographic variables and lifestyle factors and body mass index were obtained. Binary logistic regression, crude and adjusted, was employed with a significance level of 5\%. The mean SBP and DBP were $111.9 \mathrm{mmHg}$ and $69.9 \mathrm{mmHg}$, respectively. The prevalence of high BP levels was $12.4 \%$. The multivariable analysis revealed that males (OR: 2.37, 95\% CI: 1.45, 3.90), adolescents with maternal education $\leq 8$ years (OR: $1.84,95 \%$ CI: 1.03, 3.30) and overweight (OR: 3.79, 95\% CI: $2.23,6.43)$ had greater indices of high BP. The interaction term between males and overweight represented the subgroup with greater indices of high BP (OR: 6.41, 95\% CI: 3.00, 13.16). High BP affects adolescents from Ponta Grossa, State of Parana, and the groups with greater indices of high BP were males, with low maternal educational level and overweight.
\end{abstract}

Key words Hypertension, Health behavior, Adolescent, Adolescent health, Cross-sectional studies
Resumo Objetivou-se verificar a prevalência e os fatores associados à pressão arterial (PA) elevada em adolescentes de um município Brasileiro. Estudo transversal, conduzido em 653 adolescentes (57,9\% do sexo feminino) de 14 a 19 anos de idade, estudantes do ensino médio. A Pressão arterial sistólica (PAS) e a diastólica (PAD) foram mensuradas em todos os sujeitos. Variáveis sociodemográficas, do estilo de vida e indice de massa corporal foram obtidas. Regressão logística binária, bruta e ajustada, foi empregada com nível de significância de 5\%. A média da PAS e PAD foi de 111,9 $\mathrm{mmHg}$ e 69,9 $\mathrm{mmHg}$, respectivamente. A prevalência de PA elevada foi de 12,4\%. Na análise multivariável foi identificado que jovens do sexo masculino (OR: 2,37; IC95\%: 1,45-3,90), com escolaridade materna de até oito anos (OR: 1,84; IC95\%: 1,03-3,30) e com excesso de peso (OR: 3,79; IC95\%: 2,23-6,43), apresentaram maiores chances de PA elevada. O termo de interação entre sexo masculino e excesso de peso representou o subgrupo com maiores chances de PA elevada (OR: 6,41; IC95\%: 3,00-13,16). Níveis pressóricos elevados acometem adolescentes da cidade de Ponta Grossa, Paraná, e os grupos com maiores chances de PA elevada foram os do sexo masculino, com escolaridade materna baixa e com excesso de peso.

Palavras-chave Hipertensão, Conduta de saúde, Adolescente, Saúde do adolescente, Estudos transversais 


\section{Introdução}

A hipertensão arterial (HA) é uma das principais causas de doenças cardiovasculares e de mortalidade prematura em todo o mundo ${ }^{1}$. Observa-se um aumento da prevalência de $\mathrm{HA}$ em diversas faixas etárias, o que reflete em uma crescente preocupação dos órgãos de saúde pública na identificação do avanço deste quadro em grandes populações. No entanto, identificar a HA em estudos epidemiológicos é difícil, devido à operacionalização de sua mensuração (entre três e quatro visitas); assim, as medidas dos níveis pressóricos elevados apenas em um momento têm sido utilizadas como alternativa do monitoramento da PA em adolescentes ${ }^{2-4}$.

Entre os adolescentes, a prevalência de HA está em torno de $5 \%{ }^{2}$. Em países desenvolvidos, como os Estados Unidos, são relatadas prevalências de pressão arterial (PA) elevada de aproximadamente $20 \%{ }^{3}$. Em países em desenvolvimento, como o Brasil, os valores oscilam de $4 \%$ a $20 \%^{4-8}$. Estas discrepâncias nos valores da prevalência de HA podem decorrer de diferentes tipos de aparelho para mensuração da PA, como por exemplo, o uso de esfigmomanômetro de coluna de mercúrio, digitais, manuais, que podem ser colocados no braço ou no pulso. Embora tais aparelhos apresentem validade para mensuração da PA, investigações já relataram que eles podem subestimar ou superestimar os valores de PA a depender da população investigada9.

Ademais, as discrepâncias na prevalência de HA entre as populações podem está relacionadas às condições de vida, como situação socioeconômica e acesso aos serviços de saúde, e também aos hábitos de vida, relacionados à alimentação, tabagismo, uso de álcool, atividade física, estresse e obesidade, o que pode diferir de uma região para outra ${ }^{10,11}$.

A literatura demonstra que o aumento da PA na infância e na adolescência está relacionado à HA na idade adulta ${ }^{12}$. Além disso, o aumento da $\mathrm{PA}$ em idades precoces também é associado à hipertrofia ventricular esquerda, e ao maior risco de desenvolvimento da síndrome metabólica, que acelera o aparecimento de doença cardiovascular ${ }^{913}$.

Os aspectos correlatos da PA elevada em qualquer faixa etária podem ser genéticos e ambientais. O componente genético que resultaria na manifestação clínica de HA está associado à ocorrência de alterações em genes relacionados a complexos sistemas, como o transporte de eletrólitos, os mecanismos de controle simpático e endócrino ${ }^{10}$. Já os fatores ambientais são igualmente importantes na determinação da PA ao longo da vida. Fatores ambientais relacionados ao estilo de vida são responsáveis pelo aumento dos níveis pressóricos em adolescentes ${ }^{10}$. Além destes fatores, aspectos econômicos, como baixa escolaridade e baixa renda, que dificultam o acesso a serviços de saúde, têm alguma relação com a PA elevada ${ }^{11}$.

Tendo em vista a necessidade de monitorar os níveis pressóricos dos jovens, em diferentes localidades do Brasil, para aprimorar estratégias de intervenção para o combate da HA, o presente estudo tem como objetivo verificar os fatores associados à PA elevada em adolescentes do município de Ponta Grossa, Paraná, Brasil.

\section{Métodos}

\section{Local do estudo}

O presente estudo, de delineamento transversal, base escolar, foi realizado na cidade de Ponta Grossa, Paraná, Brasil, no ano de 2008 e contou com uma população de 6.597 estudantes da rede pública de ensino. Ponta Grossa situa-se no centro do estado do Paraná, que tem população de aproximadamente 311.000 habitantes $^{14}$, e um índice de desenvolvimento humano (IDH) elevado de $0,804^{15}$.

\section{População e amostra}

O processo de amostragem foi realizado por conglomerados em dois estágios. Inicialmente, todas as escolas públicas foram listadas e agrupadas de acordo com sua localização geográfica (norte, sul, leste, oeste e centro). Nove escolas foram então selecionadas como unidades primárias de amostragem, adotando a estratificação pela região geográfica, o que garantiu a representatividade das zonas geográficas da cidade na amostra. As turmas em cada escola foram as unidades secundárias de amostragem. O número de turmas selecionadas em cada escola foi definido de modo a alcançar a representatividade percentual de sua área geográfica em relação ao município como um todo.

Para o cálculo do tamanho da amostra, levou-se em consideração prevalência desconhecida para o desfecho (50\%), erro tolerável de cinco pontos percentuais, nível de confiança de $95 \%$, efeito de delineamento de 2,0, acrescentando $10 \%$ para possíveis perdas e recusas. A amostra foi estimada em 799 indivíduos. 
Definiram-se como indivíduos elegíveis para o estudo aqueles matriculados na rede estadual de ensino, que encontravam-se na sala de aula no dia da coleta e possuíssem de 14 a 19 anos de idade. Os critérios de exclusão adotados foram: (a) idade inferior a 14 anos e/ou superior ao estabelecido; (b) adolescentes que relataram ter problemas cardiovasculares prévios; (c) adolescentes grávidas ou que tiveram filho nos últimos seis meses. Considerou-se recusa o adolescente que não quis participar da pesquisa. Foi considerada perda amostral não preencher completamente o questionário.

\section{Procedimentos para coleta de dados}

O trabalho de campo foi realizado por acadêmicos do curso de enfermagem, treinados para realizar todos os procedimentos necessários, de modo a padronizar a coleta dos dados. Esta teve duração de quatro meses e foi desenvolvida nas próprias escolas.

\section{Variável dependente}

A PAS e a PAD foram avaliadas por meio do método indireto com técnica auscultatória com uso de esfigmomanômetro de coluna de mercúrio de mesa RD121 - Unitec, com escala de 0 a $300 \mathrm{mmHg}$. Os aparelhos de coluna de mercúrio são recomendados pela literatura e utilizados como método referência para validação de monitores automáticos e/ou semiautomáticos ${ }^{9}$. Para a aferição da medida, seguiram-se as recomendações das Diretrizes Brasileiras de Hipertensão Arterial $^{9}$, na qual o avaliado foi explicado sobre os procedimentos e estava em repouso por pelo menos cinco minutos em ambiente tranquilo e sem ruído, com a bexiga esvaziada, não tendo realizado exercícios físicos 90 minutos antes, nem fumado ou ingerido alimentos, café, bebidas alcoólicas e chimarrão pelo menos 30 minutos antes da entrevista. $\mathrm{O}$ ambiente era tranquilo e sem ruídos repetitivos ${ }^{9}$.

A identificação da PA elevada foi realizada da seguinte forma: 1) Para o grupo etário de 14 a 17 anos, considerou-se hipertensão arterial quando a PAS ou PAD foi igual ou maior que a encontrada no Percentil 95 (P95) das tabelas de referência do The fourth report on the diagnosis, evaluation, and treatment of high blood pressure in children and adolescents ${ }^{13}$, que varia conforme a idade e o sexo correspondentes, ajustado para o percentil de estatura do avaliado; 2) para os alunos com idades entre 18 e 19 anos foram consi- derados, como parâmetro, os valores admitidos para adultos jovens, PAS $\geq 140 \mathrm{mmHg}$ e/ou PAD $\geq 90 \mathrm{mmHg}^{9}$. A PA foi aferida duas vezes, adotando intervalo de um minuto, e foi considerada, para fins de análise, a média entre a primeira e a segunda medida.

\section{Variáveis independentes}

As informações sociodemográficas foram coletadas por meio de um questionário autoadministrado em sala de aula. As seguintes variáveis foram coletadas: sexo (masculino; feminino); idade, de forma contínua e posteriormente categorizada em 14-15, 16, 17-19 anos; escolaridade materna e paterna ( $\leq 8$ anos; $>8$ anos); o nível econômico foi identificado pelo questionário da Associação Brasileira de Empresas de Pesquisa ${ }^{16}$, que divide a população brasileira em cinco classes econômicas, por ordem decrescente de poder de compra (“A”, “B”, “C”, “D” e “E”). No presente estudo, agruparam-se as categorias " $\mathrm{A}$ " + "B", considerado estrato econômico alto; " $\mathrm{C}$ " + "D”, considerado estrato econômico baixo, conforme recomendações da literatura ${ }^{17}$. Nenhum aluno foi classificado na classe "E".

As informações sobre o nível de atividade física foram obtidas pelo questionário internacional de atividade física (IPAQ), versão curta, validado para adolescentes brasileiros ${ }^{18}$. Foram considerados pouco ativos os indivíduos que praticavam atividades físicas de intensidade moderada e/ou vigorosa por um tempo menor que 300 minutos por semana, segundo a recomendação da atividade física proposta para adolescentes ${ }^{19}$. Por outro lado, os ativos fisicamente teriam que praticar tais atividades por um período igual ou superior a 300 minutos por semana.

O comportamento sedentário foi verificado pelo tempo assistindo à televisão ou em frente ao computador. Considerou-se como comportamento sedentário de risco o tempo de assistência à televisão mais de 2 horas/dia, de acordo com recomendações internacionais ${ }^{20}$.

Para a avaliação do estado nutricional, as medidas de massa corporal e estatura foram mensuradas conforme procedimentos padronizados $^{21}$. Foi utilizada uma balança digital marca Plenna, com resolução de 100 gramas, e um estadiômetro de parede, com resolução de $1 \mathrm{~cm}$. As coletas foram feitas por dois pesquisadores treinados para a realização das medidas com erro técnico de medida $<1 \%$, considerados com níveis adequados para a realização de mensurações antropométricas ${ }^{22}$. O índice de massa cor- 
poral (IMC) foi calculado, e foram considerados os pontos de corte de Conde e Monteiro ${ }^{23}$ para IMC em adolescentes, que varia conforme a idade e sexo. Essa variável foi dicotomizada em eutrófico (baixo peso + peso normal) e excesso de peso (sobrepeso + obesidade).

\section{Análise estatística}

As análises foram realizadas no programa Stata 11.0. Empregou-se a estatística descritiva (média, desvio padrão, frequências) e inferencial. O teste qui-quadrado de heterogeneidade foi empregado para analisar as diferenças na proporção de PA elevada entre os subgrupos. O teste " $\mathrm{t}$ " para amostras independentes e a Análise de Variância "one way" foram usados para analisar as diferenças dos valores médios de PAS e PAD entre os diferentes subgrupos. Nas análises bivariadas e multivariadas para identificar os fatores associados à PA elevada empregaram-se os testes de Wald e a regressão logística binária, estimando odds ratio e IC95\%. As análises de regressão seguiram um modelo de determinação hierarquizado, hipoteticamente temporal, de acordo com as recomendações de Victora et al. ${ }^{24}$. A modelagem estatística seguiu a divisão em três blocos de variáveis: a) distal, no qual foram incluídas as variáveis sociodemográficas (sexo, idade, estrato econômico, escolaridade materna e paterna); b) intermediário, no qual foram incluídos comportamentos relacionados à saúde (atividade física e comportamento sedentário); c) proximal, no qual foi incluído o estado nutricional. Todas as variáveis foram para a análise ajustada, independentemente do p-valor na análise bruta.

$\mathrm{Na}$ análise ajustada, foi adotada abordagem hierárquica, seguindo o procedimento backward. Recorreu-se inicialmente ao ajustamento das variáveis do bloco distal para os demais fatores do mesmo nível. Em seguida, as variáveis do bloco intermediário foram controladas para as variáveis do mesmo nível e para aquelas do nível distal que permaneceram no modelo. Finalmente, na análise da variável do bloco proximal, recorreu-se ao ajustamento para os demais fatores dos níveis intermediário e distal, que permaneceram no modelo. Adotou-se como critério para permanência do fator nas análises de regressão ajustada um p-valor inferior a $0,20^{25}$. Ao final, consideraram-se significativamente associados ao desfecho sob análise aqueles fatores cujo pvalor foi inferior a 0,05 . Adicionalmente, a variável sexo foi testada como possível fator de interação com as outras variáveis independentes.

\section{Procedimentos éticos}

Os procedimentos adotados durante a realização do estudo foram aprovados pelo Comitê de Ética em Pesquisa com Seres Humanos da Universidade Estadual de Ponta Grossa, Paraná, Brasil. Todos os sujeitos que participaram do estudo entregaram o Termo de Consentimento Livre e Esclarecido assinado pelos pais/responsáveis (indivíduos com idade $<18$ anos) ou por eles mesmos (indivíduos com idade $\geq 18$ anos).

\section{Resultados}

A taxa de resposta do estudo foi de $81,7 \%$ ( $\mathrm{n}=$ 653). Houve uma maior proporção de adolescentes do sexo feminino (57,9\%), na faixa etária de 14-15 anos, de estrato econômico baixo, com escolaridade materna e paterna de até oito anos, ativos fisicamente, com comportamento sedentário de risco à saúde e eutróficos (Tabela 1).

A prevalência de PA elevada em todo o grupo foi de 12,4\% (IC95\%: 9,8-14,9). Os adolescentes do sexo masculino, com escolaridade materna $\leq 8$ anos e com excesso de peso apresentaram maiores prevalências de PA elevada. A variável idade, nível econômico, escolaridade paterna, nível de atividade física e comportamento sedentário não se apresentaram associadas com a PA elevada, indicando prevalências similares entre os subgrupos de adolescentes destas variáveis (Tabela 1).

A média da PAS e da PAD foi de $111,9 \mathrm{mmHg}$ (IC95\%: 110,9-112,9) e 69,9 mmHg (IC95\%: 69,170,7), respectivamente. A PAS foi quase $10 \mathrm{mmHg}$ maior nos adolescentes do sexo masculino em comparação ao feminino. Em relação à PAD, os estudantes do sexo masculino apresentaram mais de três mmHg do que o sexo feminino. Houve uma relação direta entre a idade e os valores pressóricos de PAS e PAD, indicando que os mais velhos apresentaram, respectivamente, valores de PAS e PAD, aproximadamente cinco e quatro $\mathrm{mmHg}$ a mais do que os adolescentes mais jovens. Adolescentes cuja escolaridade materna foi baixa apresentaram maiores valores de PAS em comparação aos jovens cuja escolaridade da mãe foi maior que oito anos. Adolescentes com excesso de peso apresentaram, respectivamente, 18 e quatro $\mathrm{mmHg}$ a mais nos valores de PAS e PAD do que os de peso normal. Em relação ao nível econômico, escolaridade paterna, nível de atividade física e comportamento sedentário, os valores de PAS e PAD foram semelhantes nos 
Tabela 1. Distribuição da amostra em relação às variáveis independentes e prevalência de pressão arterial elevada em adolescentes. Ponta Grossa, Paraná, Brasil.

\begin{tabular}{|c|c|c|c|}
\hline \multirow[b]{2}{*}{ Variáveis } & \multicolumn{2}{|c|}{ Amostra } & \multirow{2}{*}{$\begin{array}{c}\text { Pressão arterial elevada } \\
\%(\mathrm{IC} 95 \%)\end{array}$} \\
\hline & $\mathbf{n}$ & $\%($ IC95\%) & \\
\hline Total & 653 & 100 & $12,4(9,8-14,9)$ \\
\hline Sexo & & & $\mathrm{p}<0,001^{* *}$ \\
\hline Feminino & 378 & $57,9(54,0-61,6)$ & $7,9(5,1-10,7)$ \\
\hline Masculino & 275 & $42,1(38,3-45,9)$ & $18,5(13,9-23,1)$ \\
\hline Idade (anos) & & & $\mathrm{p}=0,095$ \\
\hline $14-15$ & 262 & $40,1(36,3-43,8)$ & $10,3(6,5-14,0)$ \\
\hline 16 & 214 & $32,8(29,1-36,3)$ & $11,2(6,9-15,4)$ \\
\hline $17-19$ & 177 & $27,1(23,6-30,5)$ & $16,9(11,3-22,5)$ \\
\hline Nível econômico* & & & $\mathrm{p}=0,615$ \\
\hline Alto & 256 & $47,9(43,6-52,1)$ & $12,8(8,7-17,0)$ \\
\hline Baixo & 279 & $52,1 \quad(47,9-56,3)$ & $11,4(7,7-15,2)$ \\
\hline Escolaridade Materna & & & $\mathrm{p}=0,010^{* *}$ \\
\hline$\leq 8$ anos & 424 & $66,9(63,3-70,6)$ & $14,8(11,4-18,2)$ \\
\hline$>8$ anos & 209 & $33,1 \quad(29,3-36,6)$ & $7,6(4,0-11,2)$ \\
\hline Escolaridade Paterna & & & $\mathrm{p}=0,730$ \\
\hline$\leq 8$ anos & 419 & $69,5(65,8-73,1)$ & $12,4(9,2-15,5)$ \\
\hline$>$ 8anos & 184 & $30,5(26,8-34,1)$ & $11,4(6,7-16,1)$ \\
\hline Nível de atividade física & & & $\mathrm{p}=0,760$ \\
\hline Ativo & 568 & $92,3(90,2-94,4)$ & $12,1(9,4-14,8)$ \\
\hline Pouco ativo & 47 & $7,7(5,5-9,7)$ & $10,6(1,4-19,8)$ \\
\hline Comportamento sedentário & & & $\mathrm{p}=0,931$ \\
\hline$\leq 2 \mathrm{~h} / \mathrm{dia}$ & 237 & $36,7(32,9-40,4)$ & $12,2(8,0-16,4)$ \\
\hline$>2 \mathrm{~h} / \mathrm{dia}$ & 409 & $63,3(59,5-67,0)$ & $12,4(9,2-15,6)$ \\
\hline Estado nutricional & & & $\mathrm{p}<0,001^{* *}$ \\
\hline Normal & 531 & $82,1(79,1-85,0)$ & $8,6(6,2-11,1)$ \\
\hline Excesso de peso & 116 & $17,9(14,9-20,8)$ & $27,5(19,3-35,8)$ \\
\hline
\end{tabular}

IC $=$ intervalo de confiança; ${ }^{*}$ variável com maior número de respostas ignoradas $(\mathrm{n}=118) ;{ }^{* *} \mathrm{p}<0,05$ (Teste Qui-Quadrado de heterogeneidade).

subgrupos de adolescentes destas variáveis (Tabela 2).

Na Tabela 3 verifica-se a distribuição da amostra e os valores médios de PAS e PAD dos adolescentes que apresentaram PA elevada de acordo com as variáveis independentes. A média da PAS e PAD foi de 132,7 mmHg (IC95\%: 130,4135,0) e 83,1 mmHg (IC95\%: 80,6-85,6), respectivamente. Os adolescentes do sexo masculino apresentaram cinco $\mathrm{mmHg}$ a mais nos valores de PAS do que o feminino. Houve uma relação direta entre a idade e os valores pressóricos de $\mathrm{PAD}$, indicando que os mais velhos apresentaram, aproximadamente, oito $\mathrm{mmHg}$ a mais do que os adolescentes mais jovens. Para as demais variáveis os valores de PAS e PAD foram semelhantes nos subgrupos de adolescentes que apresentaram PA elevada.
$\mathrm{Na}$ análise bruta foi verificado que os adolescentes do sexo masculino, de 17-19 anos de idade, com escolaridade materna de até oito anos, e com excesso de peso foram os grupos com maiores chances de PA elevada. Após ajuste, foi identificado que os jovens do sexo masculino (OR: 2,37; IC95\%: 1,45-3,90), com escolaridade materna de até oito anos (OR: 1,84; IC95\%: 1,033,30 ) e com excesso de peso (OR: 3,79; IC95\%: 2,23-6,43) apresentaram maiores chances de PA elevada. A variável idade teve a categoria de 17-19 anos no limiar de associação com PA elevada (OR: 1,75; IC95\%: 1,00-3,12; $\mathrm{p}=0,06)$. O único fator de interação que se mostrou associado ao desfecho foi o sexo masculino e o excesso de peso, indicando que adolescentes nesta situação apresentaram 6,41 vezes mais chances de PA elevada (Tabela 4). 
Tabela 2. Valores médios e intervalos de confiança da pressão arterial sistólica e diastólica de acordo com variáveis independentes considerando toda a amostra de adolescentes. Ponta Grossa, Paraná, Brasil.

\begin{tabular}{|c|c|c|}
\hline Variáveis & $\begin{array}{c}\text { PAS }(\mathrm{mmHg}) \\
\bar{x}(\mathrm{IC} 95 \%)\end{array}$ & $\begin{array}{c}\text { PAD }(\mathrm{mmHg}) \\
\bar{x}(\mathrm{IC} 95 \%)\end{array}$ \\
\hline Total & $111,9(110,9-112,9)$ & $69,9(69,1-70,7)$ \\
\hline Sexo & $\mathrm{p}<0,001^{*}$ & $\mathrm{p}<0,001^{*}$ \\
\hline Feminino & $108,2(107,0-109,3)$ & $68,6(67,6-69,6)$ \\
\hline Masculino & $117,1(115,4-118,6)$ & $71,7(70,4-72,9)$ \\
\hline Idade (anos) & $\mathrm{p}<0,001^{* *}$ & $\mathrm{p}<0,001^{* *}$ \\
\hline $14-15$ & $110,2(108,8-111,6)$ & $68,5(67,4-69,6)$ \\
\hline 16 & $111,5(109,7-113,3)$ & $69,4(67,9-70,8)$ \\
\hline $17-19$ & $115,0(112,9-117,0)$ & $72,6(70,9-64,2)$ \\
\hline Nível econômico* & $\mathrm{p}=0,292$ & $\mathrm{p}=0,155$ \\
\hline Alto & $112,2(110,6-113,8)$ & $70,2(69,0-71,4)$ \\
\hline Baixo & $111,0(109,5-112,5)$ & $68,9(67,7-70,1)$ \\
\hline Escolaridade Materna & $\mathrm{p}=0,048^{*}$ & $\mathrm{p}=0,245$ \\
\hline$\leq 8$ anos & $112,7(111,4-114,0)$ & $70,2(69,2-71,2)$ \\
\hline$>8$ anos & $110,5(108,9-112,1)$ & $69,2(67,8-70,6)$ \\
\hline Escolaridade Paterna & $p=0,696$ & $\mathrm{p}=0,904$ \\
\hline$\leq 8$ anos & $111,6(110,4-112,9)$ & $69,6(68,6-70,5)$ \\
\hline$>8$ anos & $112,1(110,2-114,0)$ & $69,7(68,0-71,3)$ \\
\hline Nível de atividade física & $p=0,679$ & $\mathrm{p}=0,358$ \\
\hline Ativo & $111,5(110,4-112,6)$ & $69,9(69,0-70,8)$ \\
\hline Pouco ativo & $112,3(109,0-115,6)$ & $68,5(65,8-71,1)$ \\
\hline Comportamento sedentário & $\mathrm{p}=0,509$ & $\mathrm{p}=0,059$ \\
\hline$\leq 2 \mathrm{~h} /$ dia & $112,4(110,7-114,0)$ & $70,9(69,6-72,2)$ \\
\hline$>2 \mathrm{~h} / \mathrm{dia}$ & $111,7(110,4-112,9)$ & $69,3(68,2-70,3)$ \\
\hline Estado nutricional & $\mathrm{p}<0,001^{*}$ & $\mathrm{p}<0,001^{*}$ \\
\hline Normal & $110,4(109,4-111,5)$ & $69,1 \quad(68,2-69,9)$ \\
\hline Excesso de peso & $118,1(115,6-120,5)$ & $73,0(71,0-75,0)$ \\
\hline
\end{tabular}

IC = intervalo de confiança; ${ }^{*}$ variável com maior número de respostas ignoradas $(\mathrm{n}=118) ;$ ** $\mathrm{p}<0,05$ (Teste Qui-Quadrado de heterogeneidade).

\section{Discussão}

A prevalência de PA elevada encontrada nos adolescentes do presente estudo $(12,4 \%)$ demonstra que, dependendo da localidade do Brasil, tais prevalências divergem entre os jovens ${ }^{4-8}$. Na região metropolitana de Recife, Pernambuco, foi relatada prevalência de $17,3 \%{ }^{4}$; em Maceió, Alagoas, 9,4\% ${ }^{7}$; Aracaju, Sergipe, $15 \%{ }^{8}$; na cidade de Belo Horizonte, Minas Gerais, aproximadamente $10 \%{ }^{5}$; em Londrina, Paraná, 18,6\% ${ }^{6}$. Em relação a estudos internacionais, os dados também divergem. Em Alexandria, no Egito, pesquisadores relataram que $4 \%$ dos adolescentes de 11 a 19 anos apresentaram PA elevada ${ }^{26}$; em Nova York, Estados Unidos, a prevalência foi de $26,4 \%$ dos jovens de 13 a 19 anos $^{3}$; e, em Portugal, 12,8\% dos jovens de 4 a 18 anos $^{27}$. As discrepâncias nesses valores podem ser atribuídas aos diferentes pontos de corte utilizados em diversas pesquisas, diferentes faixas etárias investigadas nos estudos e o número de aferições da PA (uma ou duas aferições).

Neste estudo, observou-se que os adolescentes do sexo masculino apresentaram maior prevalência de PA elevada do que os do sexo feminino. A literatura corrobora os valores encontra$\operatorname{dos}^{3,4,6,8}$. Em homens adultos, maiores níveis pressóricos são decorrentes do estilo de vida inadequado, como a ingestão alimentar com alto teor de sal. Contudo, em adolescentes, não se sabe ao certo a que se podem atribuir os elevados níveis pressóricos em jovens do sexo masculino, porém os hábitos alimentares são uma variável que pode interferir e que necessita ser investigada em estudos dessa natureza.

A variável idade mostrou-se associada a elevados valores de PAS e PAD em ambos os sexos, indicando que houve uma tendência de aumento dos níveis pressóricos com o passar da idade. Outros estudos com adolescentes encontraram 
Tabela 3. Distribuição da amostra e valores médios e intervalos de confiança da pressão arterial sistólica e diastólica de adolescentes que apresentaram pressão arterial elevada. Ponta Grossa, Paraná, Brasil.

\begin{tabular}{|c|c|c|c|c|}
\hline \multirow[b]{2}{*}{ Variáveis } & \multicolumn{2}{|r|}{ Amostra } & \multirow{2}{*}{$\begin{array}{c}\text { PAS }(\mathrm{mmHg}) \\
\bar{x}(\mathrm{IC} 95 \%)\end{array}$} & \multirow{2}{*}{$\begin{array}{c}\text { PAD }(\mathrm{mmHg}) \\
\bar{x}(\mathrm{IC} 95 \%)\end{array}$} \\
\hline & n & $\%($ IC95\%) & & \\
\hline Total & 81 & $12,4(9,8-14,9)$ & $132,7(130,4-135,0)$ & $83,1 \quad(80,6-85,6)$ \\
\hline Sexo & & & $\mathrm{p}=0,041^{* *}$ & $\mathrm{p}=0,447$ \\
\hline Feminino & 30 & $37,0(26,3-47,8)$ & $129,7(125,3-134,0)$ & $84,3(79,9-88,7)$ \\
\hline Masculino & 51 & $63,0(52,2-73,7)$ & $134,5(131,9-137,1)$ & $82,3(79,2-85,4)$ \\
\hline Idade (anos) & & & $\mathrm{p}=0,061$ & $\mathrm{p}=0,025^{* * *}$ \\
\hline $14-15$ & 27 & $33,3(22,8-43,8)$ & $128,9(124,7-133,0)$ & $78,5(75,1-81,9)$ \\
\hline 16 & 24 & $29,6(19,4-39,7)$ & $134,6(130,9-138,3)$ & $84,2(79,0-89,3)$ \\
\hline $17-19$ & 30 & $37,1(26,2-47,7)$ & $134,7(130,6-138,7)$ & $86,3(82,0-90,6)$ \\
\hline Nível econômico* & & & $\mathrm{p}=0,968$ & $\mathrm{p}=0,224$ \\
\hline Alto & 33 & $50,8(38,2-63,3)$ & $133,0(130,3-135,7)$ & $82,7(79,8-85,7)$ \\
\hline Baixo & 32 & $49,2(36,7-61,7)$ & $133,1(128,9-137,2)$ & $79,4(74,6-84,1)$ \\
\hline Escolaridade Materna & & & $\mathrm{p}=0,161$ & $\mathrm{p}=0,872$ \\
\hline$\leq 8$ anos & 63 & $79,8(70,7-88,8)$ & $133,5(131,2-135,7)$ & $83,0(80,1-85,8)$ \\
\hline$>8$ anos & 16 & $20,2(11,2-29,3)$ & $129,4(121,5-137,3)$ & $82,5(76,5-88,5)$ \\
\hline Escolaridade Paterna & & & $\mathrm{p}=0,463$ & $\mathrm{p}=0,905$ \\
\hline$\leq 8$ anos & 52 & $71,2(60,5-81,8)$ & $132,3(129,5-135,0)$ & $82,5(79,8-85,1)$ \\
\hline$>8$ anos & 21 & $28,8(18,1-39,4)$ & $134,3(129,1-139,4)$ & $82,9(75,8-89,9)$ \\
\hline Nível de atividade física & & & $\mathrm{p}=0,707$ & $\mathrm{p}=0,536$ \\
\hline Ativo & 69 & $93,2(87,3-99,0)$ & $132,1(129,6-134,7)$ & $83,0(80,4-85,6)$ \\
\hline Pouco ativo & 05 & $6,8(1,3-12,6)$ & $134,0(127,2-140,8)$ & $80,0(71,2-88,7)$ \\
\hline Comportamento sedentário & & & $\mathrm{p}=0,157$ & $\mathrm{p}=0,549$ \\
\hline$\leq 2 \mathrm{~h} / \mathrm{dia}$ & 29 & $36,3(25,4-47,0)$ & $130,3(125,8-134,8)$ & $84,1(80,0-88,2)$ \\
\hline$>2 \mathrm{~h} / \mathrm{dia}$ & 51 & $63,7(52,9-74,5)$ & $133,7(131,1-136,3)$ & $82,5(79,3-85,8)$ \\
\hline Estado nutricional & & & $\mathrm{p}=0,538$ & $\mathrm{p}=0,732$ \\
\hline Normal & 46 & $59,0(47,8-70,1)$ & $132,0(128,4-135,4)$ & $83,0(79,7-86,3)$ \\
\hline Excesso de peso & 32 & $41,0(30,0-52,2)$ & $133,4(130,6-136,3)$ & $82,2(78,4-85,9)$ \\
\hline
\end{tabular}

PAS - pressão arterial sistólica; PAD - pressão arterial diastólica; IC = intervalo de confiança; * variável com maior número de respostas ignoradas $(\mathrm{n}=16) ;{ }^{* *} \mathrm{p}<0,05$ (Teste “t' para amostras independentes); ${ }^{* * *} \mathrm{p}<0,05$ (Análise de variância “one way").

que à medida que aumenta a idade, os níveis pressóricos tendem a crescer ${ }^{11,12}$. A literatura reforça que, na adolescência, o aumento da idade está inversamente associado ao nível de atividade física e diretamente associado com o comportamento sedentário ${ }^{28}$. No presente estudo, obtevese que adolescentes mais velhos apresentaram menores níveis de atividade física e maior quantidade de horas durante a semana em comportamentos sedentários (dados não apresentados). Portanto, pode-se inferir que à medida que aumenta a idade, alguns comportamentos relacionados à saúde tendem a ficar inadequados, e isso pode resultar em elevados níveis pressóricos.

No presente estudo, foi encontrado que adolescentes cuja escolaridade materna foi baixa apresentaram maiores chances de PA elevada. Nenhum outro estudo foi encontrado verificando a associação entre essas variáveis, o que dificulta as comparações. Em parte, a associação entre essas variáveis pode ser atribuída a aspectos econômicos, pois as famílias cuja mãe apresenta alto nível de escolaridade tendem a ser de nível econômico elevado ${ }^{17}$. A literatura reporta que a hipertensão é mais prevalente nas camadas mais pobres, e uma justificativa é o menor acesso a alimentos saudáveis, serviços de saúde e práticas de atividade física no tempo de lazer ${ }^{10,11}$.

Assim como no presente estudo, outros levantamentos relataram que adolescentes com excesso de peso apresentaram mais chances de PA elevada em comparação aos eutróficos ${ }^{7,8,26}$. Forte associação também é relatada entre obesidade abdominal e hipertensão $\operatorname{arterial}^{26}$. Esses achados demonstram que a gordura corporal é um dos principais fatores de risco para hipertensão.

A presente investigação encontrou que adolescentes do sexo masculino que tinham excesso 
Tabela 4. Odds ratio e intervalos de confiança estimados por meio da Regressão Logística Binária entre níveis pressóricos elevados e variáveis independentes em adolescentes. Ponta Grossa, Paraná, Brasil.

\begin{tabular}{|c|c|c|c|c|}
\hline \multirow{2}{*}{ Variáveis } & \multicolumn{2}{|c|}{ Análise bruta } & \multicolumn{2}{|c|}{ Análise ajustada ${ }^{\dagger}$} \\
\hline & OR (IC95\%) & p-valor & OR (IC95\%) & p-valor \\
\hline \multicolumn{5}{|l|}{$\operatorname{Sexo}^{1}$} \\
\hline Feminino & 1 & \multirow[t]{2}{*}{$<0,01^{*}$} & 1 & \multirow[t]{2}{*}{$<0,01^{*}$} \\
\hline Masculino & $2,64(1,63-4,27)$ & & $2,37(1,45-3,90)$ & \\
\hline \multicolumn{5}{|l|}{ Idade $(\text { anos })^{1}$} \\
\hline $14-15$ & 1 & \multirow{3}{*}{$0,04^{*}$} & 1 & \multirow[t]{3}{*}{0,06} \\
\hline 16 & $1,09(0,61-1,96)$ & & $1,19(0,65-2,16)$ & \\
\hline $17-19$ & $1,77(1,02-3,10)$ & & $1,75(1,00-3,12)$ & \\
\hline \multicolumn{5}{|l|}{ Nível econômico ${ }^{1}$} \\
\hline Alto & 1 & \multirow[t]{2}{*}{0,61} & 1 & \multirow[t]{2}{*}{0,81} \\
\hline Baixo & $0,87(0,52-1,47)$ & & $1,07(0,62-1,83)$ & \\
\hline \multicolumn{5}{|l|}{ Escolaridade Materna $^{1}$} \\
\hline$\leq 8$ anos & $2,10(1,18-3,74)$ & \multirow[t]{2}{*}{$0,01^{*}$} & $1,84(1,03-3,30)$ & \multirow[t]{2}{*}{$0,04^{*}$} \\
\hline$>8$ anos & 1 & & 1 & \\
\hline \multicolumn{5}{|l|}{ Escolaridade Paterna $^{1}$} \\
\hline$\leq 8$ anos & $1,10(0,64-1,88)$ & \multirow[t]{2}{*}{0,73} & $1,11(0,56-2,18)$ & \multirow[t]{2}{*}{0,75} \\
\hline$>8$ anos & 1 & & 1 & \\
\hline \multicolumn{5}{|l|}{ Nível de atividade física ${ }^{2}$} \\
\hline Ativo & 1 & \multirow[t]{2}{*}{0,76} & 1 & \multirow[t]{2}{*}{0,94} \\
\hline Pouco ativo & $0,86(0,32-2,25)$ & & $0,97(0,36-2,60)$ & \\
\hline \multicolumn{5}{|l|}{ Comportamento sedentário ${ }^{2}$} \\
\hline dH 2 h/dia & 1 & \multirow[t]{2}{*}{0,93} & 1 & \multirow[t]{2}{*}{0,88} \\
\hline$>2 \mathrm{~h} / \mathrm{dia}$ & $1,02(0,62-1,66)$ & & $1,04(0,62-1,74)$ & \\
\hline \multicolumn{5}{|l|}{ Estado nutricional $^{3}$} \\
\hline Normal & 1 & \multirow[t]{2}{*}{$<0,01^{*}$} & 1 & \multirow[t]{2}{*}{$<0,01^{*}$} \\
\hline Excesso de peso & $4,01(2,41-6,67)$ & & $3,79(2,23-6,43)$ & \\
\hline \multirow{2}{*}{\multicolumn{3}{|c|}{$\begin{array}{l}\text { Sexo x Estado nutricional } \\
\text { Masculino x Excesso de peso }\end{array}$}} & & \\
\hline & & & $6,41(3,00-13,16)$ & $<0,01^{*}$ \\
\hline
\end{tabular}

Nota: Números sobrescritos 1,2,3 - ordem de entrada das variáveis no modelo ajustado.

OR = odds ratio; IC95\% = intervalo de confiança de $95 \% ;{ }^{*} \mathrm{p} \leq 0,05$; $\dagger$ - variáveis ajustadas por todas as variáveis, independente do p-valor na análise bruta.

de peso foi o subgrupo populacional com maiores chances de PA elevada. Este achado vai ao encontro da literatura que destaca o sexo masculino como mais susceptível a PA elevada ${ }^{3,4}$, assim como também as pessoas com excesso de peso ${ }^{7,8}$. Entretanto, o que ficou evidente no presente estudo foi que, ser do sexo masculino representou, aproximadamente, duas vezes mais chances de PA elevada, e ter excesso de peso correspondia a 3,79 vezes mais chances de PA elevada. Porém, quando as duas variáveis foram analisadas conjuntamente (interação), os valores de odds ratio aumentaram para 6,41. Neste sentido, estratégias de combate a PA elevada e ao excesso de peso devem ser direcionadas, especialmente aos estudantes do sexo masculino da cidade de Ponta Grossa.

A literatura evidencia que aspectos do estilo de vida, como o nível de atividade física e o com- portamento sedentário, estão relacionados, independentemente, com o excesso de gordura corporal ${ }^{29,30}$. Ou seja, o sujeito pode atender as recomendações para a prática de atividade física, porém apresentar comportamento sedentário de risco e vice-versa ${ }^{31}$, o que pode acarretar na vida adulta doenças e agravos à saúde, como a obesidade e a hipertensão arterial sistêmica9 ${ }^{9}$. Esperava-se encontrar associação entre as variáveis do estilo de vida e PA elevada, porém tal resultado não foi observado. Alguns aspectos podem justificar esta falta de associação, como por exemplo, o desenho do estudo, de caráter transversal que impede o acompanhamento da exposição e do desfecho. Além disso, a população do presente estudo é formada por jovens e os efeitos de um estilo de vida insuficientemente ativo e sedentário começam a aparecer com mais evidência a 
partir da meia idade ${ }^{29}$. De qualquer forma, mesmo que os dois comportamentos do estilo de vida não tenham se associado com os níveis pressóricos elevados estimula-se que orientações no ambiente escolar sejam dadas para a adoção e a manutenção de hábitos saudáveis por toda a vida.

O estudo apresenta limitações que necessitam ser consideradas na interpretação dos resultados. O delineamento empregado (transversal) não permite estabelecer relação de causa-efeito entre as variáveis estudadas. A causalidade reversa também não pode ser descartada. A aferição da PA em um único momento, haja vista que as Diretrizes Brasileiras de Hipertensão Arterial recomendam a aferição da PA em momentos e dias distintos para verificar se valores pressóricos elevados se mantém ${ }^{15}$. Esta situação pode ter acarretado uma superestimação na prevalência de PA elevada.

Porém, destaca-se como pontos positivos a utilização de medidas padronizadas e mensuradas de massa corporal e estatura, a utilização de um questionário válido para adolescentes na estimativa da atividade física. Além da PA ter sido mensurada com duas medidas e em um único período do dia (manhã). Estes fatores contribuem com a qualidade dos dados.

Pode-se concluir que níveis pressóricos elevados acometem adolescentes da cidade de Ponta Grossa, Paraná, sendo mais prevalente no sexo masculino. A PAS e a PAD foram maiores nos adolescentes do sexo masculino, de 17-19 anos e com excesso de peso. Adolescentes cuja mãe apresentava baixa escolaridade também tiveram valores elevados de PAS. Os grupos com maiores chances de PA elevada foram os adolescentes do sexo masculino, aqueles com escolaridade materna inferior a nove anos de estudo e com excesso de peso. Além disso, adolescentes do sexo masculino que tinham excesso de peso foi o grupo de estudantes com maiores chances de PA elevada. O estudo demonstra a necessidade de campanhas e intervenções no ambiente escolar para tornar os jovens mais saudáveis, com orientações a respeito de hábitos alimentares, redução de peso e, consequentemente, adequação dos níveis pressóricos.

\section{Colaboradores}

DAS Silva pensou o problema de pesquisa, realizou a análise estatística e contribuiu na redação do manuscrito. LRA Lima, RA Dellagrana, EDA Bacil, CR Rech contribuíram na concepção do projeto, na coleta de dados, na redação do manuscrito e na análise crítica do artigo.

\section{Referências}

1. Danaei G, Finucane MM, Lin JK, Singh GM, Paciorek CJ, Cowan MJ, Farzadfar F, Stevens GA, Lim SS, Riley LM, Ezzati M; Global Burden of Metabolic Risk Factors of Chronic Diseases Collaborating Group (Blood Pressure). National, regional, and global trends in systolic blood pressure since 1980 systematic analysis of health examination surveys and epidemiological studies with 786 country-years and 5.4 million participants. Lancet 2011; 377(9765): 568-577.

2. Falkner B. Hypertension in children and adolescents: epidemiology and natural history. Pediatr Nephrol 2010; 25(7):1219-1224.

3. Zhou P, Chaudhari RS, Antal Z. Gender differences in cardiovascular risks of obese adolescents in the bronx. J Clin Res Pediatr Endocrinol 2010; 2(2):6771.

4. Gomes BMR, Alves JGB. Prevalência de hipertensão arterial e fatores associados em estudantes de Ensino Médio de escolas públicas da Região Metropolitana do Recife, Pernambuco, Brasil, 2006. Cad Saude Publica 2009; 25(2):375-381.

5. Oliveira RG, Lamounier JA, Oliveira ADB, Castro MDR, Oliveira JS. Pressão arterial em escolares e adolescentes - O estudo de Belo Horizonte. J Pediatr 1999; 75(4):256-266. 
6. Romanzini M, Reichert FF, Lopes AS, Petroski EL, Farias Júnior JC. Prevalência de fatores de risco cardiovascular em adolescentes. Cad Saude Publica 2008; 24(11):2573-2581.

7. Moura AA, Silva MAM, Ferraz MRMT, Rivera IR. Prevalência de pressão arterial elevada em escolares e adolescentes de Maceió. J Pediatr 2004; $80(1): 35-40$.

8. Polderman J, Gurgel RQ, Barreto-Filho JA, Roelofs $\mathrm{R}$, Ramos RE, de Munter JS et al. Blood pressure and BMI in adolescents in Aracaju, Brazil. Public Health Nutr 2011; 14(6):1064-1070.

9. Sociedade Brasileira de Hipertensão, Sociedade Brasileira de Cardiologia e Sociedade Brasileira de Nefrologia. V Diretrizes Brasileiras de Hipertensão Arterial. Sociedade Brasileira de Hipertensão, Sociedade Brasileira de Cardiologia e Sociedade Brasileira de Nefrologia. Arq Bras Cardiol 2007; 89:e24-e79.

10. Santos ZMSA, Caetano JA, Moreira FGA. Atuação dos pais na prevenção da hipertensão arterial: uma tecnologia educativa em saúde. Cien Saude Colet 2011; 16(11):4385-4394.

11. Ferreira JS, Aydos RD. Prevalência de hipertensão arterial em crianças e adolescentes obesos. Cien Saude Colet 2010; 15(1):97-104.

12. Bao W, Threefoot SA, Srinivasan SR, Berenson GS. Essential hypertension predicted by tracking of elevated blood pressure from childhood to adulthood: The Bogalusa Heart Study. Am J Hypertens 1995; 8(7):657-665.

13. National High Blood Pressure Education Program Working Group on High Blood Pressure in Children and Adolescents. The fourth report on the diagnosis, evaluation, and treatment of high blood pressure in children and adolescents. Pediatrics 2004; 114(Supl. 2 4th Report):555-576.

14. Instituto Brasileiro de Geografia e Estatística (IBGE). $I B G E$ cidades. [homepage na internet]. [acessado 2010 out 22]. Disponível em: http://www.ibge.gov.br/ cidadesat/topwindow.htm?1.

15. Organização das Nações Unidas (ONU). Índice de Desenvolvimento Humano, Municipal 1991 e 2000. Todos os municípios do Brasil. [homepage na Internet]. [acessado 2010 mar 10]. Disponível em: http://www.pnud.org.br/atlas/ranking/IDH M\%2091\%2000\%20Ranking\%20decrescente\%20 (pelos\%20dados\%20de\%202000).htm

16. Associação Brasileira de Empresas de Pesquisa (ABEP). Critério de Classificação Econômica Brasil. 2003. [homepage na Internet]. [acessado 2005 abr 10]. Disponível em: http://www.abep.org/codigosguias/ABEP_CCEB.pdf

17. Silva DAS, Pereira IMM. Estágios de mudança de comportamento para atividade física e fatores associados em acadêmicos de Educação Física. Rev Bras Ativ Fís Saúde 2010; 15(1):15-20.

18. Guedes DP, Lopes CC, Guedes JERP. Reprodutibilidade e validade do questionário internacional de atividade física em adolescentes. Rev Bras Med Esporte 2005; 11(2):151-158.

19. Strong WB, Malina RM, Blimkie CJ, Daniels SR, Dishman RK, Gutin B, Hergenroeder AC, Must A, Nixon PA, Pivarnik JM, Rowland T, Trost S, Trudeau F. Evidence based physical activity for school-age youth. J Pediatr 2005; 146(6):732-737.
20. Children, adolescents, and television. American Academy of Pediatrics Committee on Communications. Pediatrics 1995; 96(4 Pt 1):786-787.

21. World Health Organization (WHO). Physical Status: the use and interpretation of anthropometry. WHO Technical Report Series no 854. Geneva: WHO; 1995.

22. Silva DAS, Pelegrini A, Pires-Neto CS, Vieira MFS, Petroski EL. O antropometrista na busca de dados mais confiáveis. Rev Bras Cineantropom Desempenho Hum 2011; 13(1):82-85.

23. Conde WL, Monteiro CA. Body mass index cutoff points for evaluation of nutritional status in Brazilian children and adolescents. J Pediatr 2006; 82(4):266-272.

24. Victora CG, Huttly SR, Fuchs SC, Olinto MT. The role of conceptual frameworks in epidemiological analisys: a hierarchical approach. Int J Epidemiol 1997; 26(1):224-227.

25. Maldonado G, Greenland S. Simulation study of confounder-selection strategies. Am J Epidemiol 1993; 138(11):923-936.

26. Abolfotouh MA, Sallam SA, Mohammed MS, Loutfy AA, Hasab AA. Prevalence of elevated blood pressure and association with obesity in egyptian school adolescents. Int J Hypertens 2011:952537.

27. Maldonado J, Pereira T, Fernandes R, Santos R, Carvalho M. An approach of hypertension prevalence in a sample of 5381 Portuguese children and adolescents. The AVELEIRA registry. "Hypertension in Children". Blood Press 2011; 20(3):153-157.

28. Silva DAS, Lima JO, Silva RJS, Prado RL. Nível de atividade física e comportamento sedentário em escolares. Rev Bras Cineantropom Desempenho Hum 2009; 11(3):299-306.

29. Reilly JJ. Physical activity, sedentary behaviour and energy balance in the preschool child: opportunities for early obesity prevention. Proc Nutr Soc 2008; 67(3):317-325.

30. Muraro AP, Santos DF, Rodrigues PRM, Braga JU. Fatores associados à Hipertensão Arterial Sistêmica autorreferida segundo VIGITEL nas 26 capitais brasileiras e no Distrito Federal em 2008. Cien Saude Colet 2013; 18(5):1387-1398.

31. Pate RR, O’Neill JR, Lobelo F. The evolving definition of "sedentary". Exerc Sport Sci Rev 2008; 36(4): 173-178.

Artigo apresentado em 25/04/2012

Aprovado em 22/06/2012

Versão final apresentada em 12/07/2012 